\title{
Association of D-Dimer Levels On Admission With Outcomes in Elderly Patients With Chronic Obstructive Pulmonary Disease Exacerbation
}

Jinling Ma ( $\triangle$ majinling1@163.com )

Chinese PLA General Hospital https://orcid.org/0000-0003-1523-5892

Chengxin Li

Chinese PLA 15 Hospital

Meng Gao

Chinese PLA General Hospital

Xiujie Wang

Zhaoyuan People's Hospital

Lei He

Dancheng People's Hospital

\section{Research Article}

Keywords: Chronic obstructive pulmonary disease, D-dimer, Elderly

Posted Date: November 10th, 2021

DOI: https://doi.org/10.21203/rs.3.rs-1030717/v1

License: (c) (i) This work is licensed under a Creative Commons Attribution 4.0 International License. Read Full License 


\section{Abstract}

Elevated D-dimer is a reliable prognostic marker for both short-term and long-term survival in patients admitted for acute exacerbation of chronic obstructive pulmonary disease (AECOPD). We retrospectively reviewed the data of consecutive patients aged 80 years and older with a primary admission diagnosis of AECOPD between December 2008 and December 2014. A total of 2206 patients were included. The patients were followed up for 30 days. D-dimer levels were higher in non-survivors than in survivors $(p<$ 0.001). Patients with need for MV had higher D-dimer levels than patients without need for $M V(p<$ 0.001). Moreover, a significant correlation between D-dimer level and C-reaction protein (CRP) was found $(r=0.2271, p<0.001)$. D-dimer level above $1.66 \mathrm{mg} / \mathrm{L}$ as the optimal cutoff level predicted 30 -day allcause mortality with a sensitivity and specificity of $87.6 \%$ and $89.1 \%$, respectively. Similarly, D-dimer above $1.33 \mathrm{mg} / \mathrm{L}$ predicted 30-day need for MV with a sensitivity and specificity of $89.4 \%$ and $82.9 \%$, respectively. Furthermore, Kaplan-Meier event-free survival curves demonstrated that higher D-dimer levels had worse event-free survival. D-dimer could be used as a valuable biomarker to predict both 30day all-cause mortality and the need for MV in elderly patients admitted for AECOPD.

\section{Introduction}

Chronic obstructive pulmonary disease (COPD), especially acute exacerbation of COPD (AECOPD), is commonly associated with systemic inflammation [1]. AECOPD is associated with substantial in-hospital mortality and may impact long-term prognosis [2]. The frequency and severity of exacerbation are the most important factors that determine the overall prognosis of COPD [3]. Heart failure and COPD coincide in a significant number of patients [4]. Especially, in the elderly with AECOPD, the classic clinical signs may be delayed, because of advanced age and comorbidity, and the mortality is increased. Early and precise individual risk assessment is crucial to estimate disease severity, clinical management and guide therapeutic options. Early identification of patients with poor clinical outcome or at risk for treatment failure may be helpful in increasing the overall cure rate, and is very important in reducing mortality through readily available laboratory tests. D-dimer is a biomarker of fibrin formation and degradation. An elevated D-dimer is independently associated with an increased risk for incident venous thromboembolism(VTE), recurrent VTE, and increased mortality $[5,6]$. High levels of D-dimer are associated with 28-day mortality in patients with infection or sepsis [7]. D-dimer greater than $1 \mu \mathrm{g} / \mathrm{mL}$ is associated with fatal outcome of coronavirus disease 2019 [8]. At the late stages of novel coronavirus pneumonia, levels of D-dimer moderately or markedly elevated in all deaths, which suggested a common coagulation activation and secondary hyperfibrinolysis condition in these patients [9]. D-dimer and Creactive protein (CRP) at admission appear to be useful biomarkers for assessing the severity of community-acquired pneumonia (CAP) [10]. CRP and D-dimer levels were related to severe 2019 novel coronavirus pneumonia and composite endpoint [11]. Elevated D-dimer is a reliable prognostic marker for both short-term and long-term survival in patients admitted for AECOPD[2]. D-dimer $>2.0 \mu \mathrm{g} / \mathrm{mL}$ were associated with in-hospital mortality in COPD-CAP patients [12]. Additionally, the frequency of abnormally 
elevated D-dimer levels is higher in older individuals even in the absence of detectable thrombosis [13, $14]$.

Limited data are available on the relationship between D-dimer levels and AECOPD in patients aged 80 years and older. In the present study, we aimed to evaluate the association between D-dimer levels and markers of inflammation (CRP) obtained upon admission in patients with AECOPD. We also determined whether D-dimer levels correlate with both 30-day all-cause mortality and the need for mechanical ventilation (MV), and ultimately to evaluate its ability to stratify AECOPD patients into different management groups.

\section{Methods}

\section{Study population}

We retrospectively reviewed the data of consecutive patients aged 80 years and older with a primary admission diagnosis of AECOPD who were admitted to the Chinese PLA General Hospital, Zhaoyuan People's Hospital, Dancheng People's Hospital, and Chinese PLA 15 Hospital between December 2008 and December 2014. The medical histories, demographic characteristics, and laboratory findings performed on admission, were obtained from the hospitals' database.

The patients were included if the following criteria were met: diagnosis of AECOPD according to the criteria set by the Global Initiative for Chronic Obstructive Lung Disease [15]. In addition, patients whose D-dimer and CRP levels were obtained at the time of admission to the hospital and prior to the commencement of antibiotic therapy were included in the study. Increase of D-dimer has been demonstrated in various clinical conditions, including trauma, malignancy, sepsis, disseminated intravascular coagulation, and acute coronary syndromes [16]. Patients with recent surgery (within the last month of index admission), recent trauma (within the last month), active cancer, previous diagnosis of confirmed pulmonary embolism (PE) and/or deep vein thrombosis (DVT), or a recent history of myocardial infarction or cerebral infarction were not included in the study. Moreover, patients who received any kind of anticoagulation within the last 3 months were excluded from the study. The study was approved by the Ethics Committees of the four hospitals.

\section{D-dimer Testing}

D-dimer was measured by a turbidimetric immunoassay using a D-DI kit (STA-Liatest). The normal values were considered below the cutoff level of $0.5 \mathrm{mg} / \mathrm{L}$. CRP was measured by an immunoturbidimetric assay. An increased level of CRP was defined as $>0.8 \mathrm{mg} / \mathrm{dL}$.

\section{Follow-up}

After admission, the patients were followed up for 30 days by review of the clinical data or a research assistant. No patients were lost during the follow-up period. The primary end point was the need for MV, and the secondary outcome was 30-day all-cause mortality. 


\section{Statistical analysis}

The categorical variables were presented as proportions (percentages). Normally distributed continuous data were expressed as mean \pm SD. Categorical variables were compared using $\chi^{2}$ tests. Logistic regression analysis was used to predict the prevalence of 30-day all-cause mortality and need for MV, with adjustments for age, sex, and co-morbidities. The results were presented as adjusted odds ratios (OR) and their $95 \%$ confidence interval $(\mathrm{Cl})$. The Spearman rank correlation test was used for analysis of the linear relationship between D-dimer levels and CRP. Receiver operating characteristic (ROC) analysis was used to determine the optimal cutoff level for D-dimer that predict 30-day all-cause mortality and need for MV. An optimal cutoff was obtained considering the combination of highest sensitivity and specificity. Survival curves were estimated by the Kaplan-Meier analysis and compared using the logrank test. All data were processed using the PASW (version 18.0; SPSS, Chicago, IL, USA). A p value $<0.05$ was considered statistically significant.

\section{Results}

A total of 2206 consecutive patients aged 80 years and over were included. During the 30-day follow-up period after admission, the overall all-cause mortality was $6.2 \%$ (137 of 2206 patients). 179 patients needed MV. Table 1 presents an overview of the clinical characteristics according to the occurrence of $30-$ day survival and the need for MV. The D-dimer levels on admission were higher in non-survivors than in survivors ( $p<0.001$, Table 1, Figure 1). The patients with need for MV had higher D-dimer levels than patients without need for MV $(p<0.001$, Table 1$)$. D-dimer levels were significantly different among the two groups (Figure 1). Moreover, age, CRP, and chronic renal insufficiency were significantly associated with worse 30-day survival and the need for MV. We also found a significant correlation between D-dimer level and CRP $(r=0.2271, p<0.001)$. 
Table 1

Clinical characteristics according to the occurrence of 30-day survival and need for MV

\begin{tabular}{|c|c|c|c|c|c|c|}
\hline & Survivors & Nonsurvivors & $\begin{array}{l}p \\
\text { value }\end{array}$ & $\begin{array}{l}\text { Need for } \\
\text { MV }\end{array}$ & $\begin{array}{l}\text { Without } \\
\text { need } \\
\text { for MV }\end{array}$ & $\begin{array}{l}p \\
\text { value }\end{array}$ \\
\hline & $n=2069$ & $n=137$ & & $n=179$ & $n=2027$ & \\
\hline Age(year) & $89.2 \pm 4.0$ & $90.4 \pm 3.9$ & 0.001 & $90.3 \pm 4.0$ & $89.2 \pm 4.0$ & $<0.001$ \\
\hline Male,n & 1594 & 99 & 0.199 & 130 & 1563 & 0.174 \\
\hline \multicolumn{7}{|l|}{ Co-morbidities, n } \\
\hline Hypertension & 1346 & 91 & 0.745 & 124 & 1313 & 0.226 \\
\hline Atrial fibrillation & 347 & 27 & 0.375 & 35 & 339 & 0.334 \\
\hline Coronary heart disease & 1669 & 108 & 0.599 & 148 & 1629 & 0.453 \\
\hline $\begin{array}{l}\text { Chronic renal } \\
\text { insufficiency }\end{array}$ & 546 & 53 & 0.002 & 64 & 535 & 0.007 \\
\hline Diabetes mellitus & 820 & 57 & 0.648 & 80 & 797 & 0.159 \\
\hline $\mathrm{CRP}(\mathrm{mg} / \mathrm{dL})$ & $1.69 \pm 3.77$ & $4.93 \pm 5.35$ & $<0.001$ & $4.92 \pm 5.16$ & $1.62 \pm 3.72$ & $<0.001$ \\
\hline D-dimer level(mg/L) & $0.88 \pm 0.69$ & $2.86 \pm 1.51$ & $<0.001$ & $2.67 \pm 1.43$ & $0.85 \pm 0.66$ & $<0.001$ \\
\hline
\end{tabular}

ROC analysis (Figure 2) identified D-dimer $1.66 \mathrm{mg} / \mathrm{L}$ on admission as the optimal cutoff level to discriminate survivors (area under the ROC $=0.9347$, standard error $=0.0107 ; 95 \% \mathrm{Cl}=0.9136-0.9559, p$ < 0.001). D-dimer level above $1.66 \mathrm{mg} / \mathrm{L}$ had a sensitivity and specificity of $87.6 \%$ and $89.1 \%$, respectively, for predicting 30-day mortality. In addition, ROC analysis (Figure 2) identified D-dimer > $1.33 \mathrm{mg} / \mathrm{L}$ as the optimal cutoff level to predict 30-day need for MV with a sensitivity and specificity of $89.4 \%$ and $82.9 \%$, respectively (area under the ROC $=0.9305$, standard error $=0.0091,95 \% \mathrm{Cl}=0.9127-$ $0.9483, p<0.001)$.

The confounding factors of age, sex, co-morbidities, and CRP were adjusted in a multivariate logistic regression analysis. This analysis showed that $\mathrm{D}$-dimer $\geq 1.66 \mathrm{mg} / \mathrm{L}(\mathrm{OR} 47.5,95 \% \mathrm{Cl}=27.94-80.67, p$ $<0.001)$ remained significantly and independently associated with 30-day mortality. Additionally, D-dimer $\geq 1.33 \mathrm{mg} / \mathrm{L}$ (OR $33.0,95 \% \mathrm{Cl}=20.19-54.04, p<0.001)$ remained significantly and independently associated with the 30-day need for MV (Table 2). The results showed that higher D-dimer levels remained an independent predictor for the development of 30-day adverse outcome. 
Table 2

Risk factors associated with 30-day survival and need for MV in a multivariate logistic regression analysis

\begin{tabular}{|lllllll|}
\hline Risk factors & \multicolumn{3}{l}{ 30-day survival } & \multicolumn{4}{l|}{ Need for MV } \\
\hline & OR & $95 \% \mathrm{Cl}$ & $p$ value & OR & $95 \% \mathrm{Cl}$ & $p$ value \\
\hline Age & 1.02 & $0.96-1.08$ & 0.527 & 1 & $0.96-1.05$ & 0.909 \\
\hline Male & 0.72 & $0.45-1.18$ & 0.192 & 0.83 & $0.55-1.26$ & 0.378 \\
\hline Co-morbidities & & & & & & \\
\hline Hypertension & 0.81 & $0.51-1.29$ & 0.368 & 1 & $0.67-1.50$ & 0.986 \\
\hline Atrial fibrillation & 1.07 & $0.62-1.85$ & 0.809 & 1.19 & $0.74-1.92$ & 0.466 \\
\hline Coronary heart disease & 0.57 & $0.33-0.99$ & 0.049 & 1.15 & $0.71-1.87$ & 0.578 \\
\hline Chronic renal insufficiency & 1.48 & $0.93-2.33$ & 0.095 & 1.02 & $0.69-1.52$ & 0.904 \\
\hline Diabetes mellitus & 1.1 & $0.71-1.71$ & 0.677 & 1.38 & $0.94-2.02$ & 0.102 \\
\hline CRP>0.8 mg/dL & 4.42 & $2.75-7.11$ & $<0.001$ & 4.74 & $3.17-7.10$ & $<0.001$ \\
\hline D-dimer $\geq 1.66$ mg/L & 47.5 & $27.9-80.7$ & $<0.001$ & 33.0 & $20.2-54.0$ & $<0.001$ \\
\hline
\end{tabular}

Kaplan-Meier event-free survival curves demonstrated that patients with D-dimer $\geq 1.66 \mathrm{mg} / \mathrm{L}$ showed worse event-free survival compared with those with $\mathrm{D}$-dimer $<1.66 \mathrm{mg} / \mathrm{L}$ during the 30 -day follow-up period $(p<0.001)$. Similarly, a significant survival disadvantage for patients with $D$-dimer level $\geq 1.33$ $\mathrm{mg} / \mathrm{L}$ was found $(p<0.001)$ (Figure 3 ).

\section{Discussion}

Our findings revealed that high D-dimer levels on admission in this study population were associated with 30-day all-cause mortality and the need for MV, and D-dimer levels above $1.66 \mathrm{mg} / \mathrm{L}$ serve as a strong independent indicator of 30-day mortality in patients with a primary admission diagnosis of AECOPD. Ddimer levels above $1.33 \mathrm{mg} / \mathrm{L}$ were also good predictors of the need for MV. This association remained after adjustment for age, sex, co-morbidities, and CRP. In addition, the values of D-dimer correlated well with the values of the CRP level.

An elevated D-dimer was associated with increased total mortality in the absence of clinically overt disease $[17,18]$, as well as among patients with PE and aortic dissection $[19,20]$. D-dimer levels also appeared to be accurate markers of the severity of severe acute pancreatitis and increased levels of Ddimer were associated with the development of organ dysfunction [21, 22]. Moreover, elevated D-dimer is associated with higher cardiovascular mortality among patients with cardiovascular disease [23-25]. Several studies have found this relationship existed in the patients with CAP and sepsis; moreover, Ddimer levels are closely related to severity of CAP [26-31]. An admission D-dimer level less than 500 
$\mathrm{ng} / \mathrm{mL}$ is also associated with low risk of short-term death and major morbidity in CAP patients [32]. In addition, D-dimer levels greater than $2,000 \mathrm{ng} / \mathrm{mL}$ identify a subgroup of CAP patients who are at increased risk for in-hospital death [26]. As a result, an elevated D-dimer is an independent correlate of increased mortality across a broad variety of disease states. Many studies have demonstrated an association between elevated D-dimer and poor prognosis, but no single cutpoint has been identified which consistently optimizes the prognostic value of the biomarker. CRP levels may also reflect the severity of inflammation[33]. In accordance with these previous finding, our results showed that increased D-dimer levels were associated with adverse outcome. D-dimer level (above $1.66 \mathrm{mg} / \mathrm{L}$ ) on admission was shown to be useful in predicting mortality, with a high sensitivity of $87.6 \%$ and negative predictive value of $89.1 \%$. Moreover, if the D-dimer level was above $1.33 \mathrm{mg} / \mathrm{L}$, a high level correlated with poor outcomes of the need for MV. Consequently, these patients may require special attention after admission. D-dimer level could be used to distinguish a subgroup of patients with increased risk for both mortality and the need for MV. The correlation between coagulation factors and adverse outcome also suggests that anticoagulant therapies may be beneficial in patients with severe AECOPD.

The mechanism underlying the elevated D-dimer levels might be complicated. Activation of the coagulation cascade is a common and early event in patients with infection [7]. Severe coagulative disorder characterized by the diffuse formation of intravascular microthrombi and activation of fibrinolysis could be the dominant cause of this phenomenon [34]. Systemic pro-inflammatory cytokine responses are mediators of atherosclerosis directly contributing to plaque rupture through local inflammation, induction of procoagulant factors, and haemodynamic changes, which predispose to ischaemia and thrombosis $[35,36]$. D-dimer levels may be elevated in AECOPD patients as an inflammatory and prothrombotic host response to infection. The mechanism underlying the increases in D-dimer levels may be the formation of multiple intravascular thrombi. Furthermore, increased inflammatory response during AECOPD may increase the ongoing inflammatory process associated with atherosclerosis, resulting in escalation of atherosclerosis and coronary artery luminal narrowing possibly through processes that involve fibrinolysis releasing D-dimer into the circulation. This phenomenon thereby increases the likelihood of atheroma plaque rupture and subsequent ischemia, as well as myocardial events [37]. A persistent prothrombotic state after infection may explain the epidemiologic link between infection and higher risk of cardiovascular disease [31]. Early elevation of D-dimer, together with other inflammatory factors, may contribute to the development or to the severity of several chronic diseases, including cardiovascular disease, leading to augmented mortality [17].The prothrombotic changes associated with acute inflammation reflected by the elevated D-dimer levels suggest that undetected thromboembolism may contribute to mortality in AECOPD patients. D-dimer may represent the summation of pro-coagulant balance or genetic factors, the extent of subclinical atherosclerosis, or the presence of underlying coagulation disorders that predispose to poor prognosis. Interventions, such as aspirin and statins, with beneficial effects on resolution of the prothrombotic state and inflammation, should be investigated to improve long-term outcomes after pneumonia [38]. Our results suggest that higher D-dimer levels may provide additional prognostic information. D-dimer is a simple severity assessment tool that is slightly better than other current scoring systems in predicting adverse outcomes, 
such as the need for MV and therapeutic failure. Measurement of the D-dimer level may have the potential of identify a subgroup of patients with COPD who are at low risk of adverse outcomes who may be suitable for outpatient therapy. This approach may also allow the selection of patients who will need a more intensive follow-up after admission, or those patients who might benefit from an antithrombotic therapy. Further prospective multiple center studies with a large number of patients are required to establish the exact role of D-dimer as a possible novel prognostic marker in AECOPD patients and form stronger conclusions.

This study has several limitations that should be considered. First, patients with elevated D-dimer levels were not systematically assessed to detect the presence of VTE. In this retrospective study, PE or DVT was ruled out by spiral CT scanning or peripheral vein compression ultrasonography only in patients with a high pretest probability. Therefore, not all VTE episode can be excluded, because of the presence of asymptomatic thrombus at the time of D-dimer measurement. Second, the overall mortality was different from that of other reports. The D-dimer cutoff values associated with mortality in the present study may be substantially different among patients, which probably reflected the fact that in our study, patients with recent surgery, recent trauma, active cancer, or a recent history of myocardial infarction or cerebral infarction were not included. Third, in our heterogeneous population, the patients were elderly, aged 80 years and older, and co-morbidities may have affected the coagulation process. Therefore, these characteristics may limit the generalizability of our findings to this age bracket. D-dimer is also influenced by other concomitant medical conditions, such as atrial fibrillation, heart failure, peripheral artery disease and renal failure [39-42]. Moreover, in this study, we did not exclude the patients exhibiting these concomitant medical conditions, which may have reduced the specificity of D-dimer in predicting mortality. Fourth, therapeutic interventions may be highly influenced by age and empirical antibiotic therapy, which may add some bias to our main results. Finally, D-dimer possesses high heritability and this fact supports the notion that D-dimer levels reflect genetic factors [43]. This study was performed in four different hospitals and most of the patients were from North China; thus, our findings may not be completely representative of the population of other locations. Moreover, D-dimer may not have the same testing characteristics when applied to other populations, and will probably perform worse. Although caution is necessary in the interpretation of our results, we consider it improbable that these limitations have influenced our main findings.

\section{Conclusion}

In conclusion, our results suggest that D-dimer is a prognostic biomarker with the potential to identify patients at low risk of 30-day mortality or the need for $\mathrm{MV}$ in patients with a primary admission diagnosis of AECOPD. The main usefulness of the D-dimer is its ability to identify patients who need more aggressive monitoring and treatment after admission. D-dimer measurement could help risk stratification in AECOPD patients.

\section{Declarations}




\section{Funding Source}

None.

\section{Disclosure}

None.

\section{Compliance with ethical standards}

Conflict of interest The authors declare that they have no conflict of interest.

Ethical approval The study was approved by the Ethics Committees of the four hospitals(Chinese PLA General Hospital, Zhaoyuan People's Hospital, Dancheng People's Hospital, and Chinese PLA 15 Hospital) and conducted in compliance with the Declaration of Helsinki.

Informed consent We retrospectively reviewed the data. Informed consent was waived due to the retrospective nature.

Funding This research did not receive any specific grant from funding agencies in the public, commercial, or not-for-profit sectors.

\section{References}

1. Tudorache E, Oancea C, Avram C, Fira-Mladinescu O, Petrescu L, Timar B. Balance impairment and systemic inflammation in chronic obstructive pulmonary disease. Int $\mathrm{J}$ Chron Obstruct Pulmon Dis. 2015; 10:1847-52.

2. Fruchter $\mathrm{O}$, Yigla M, Kramer MR. D-dimer as a prognostic biomarker for mortality in chronic obstructive pulmonary disease exacerbation. Am J Med Sci. 2015; 349(1): 29-35.

3. Groenewegen $\mathrm{KH}$, Schols AM, Wouters EF. Mortality and mortality-related factors after hospitalization for acute exacerbation of COPD. Chest. 2003; 124(2): 459-67.

4. Canepa M, Franssen FME, Olschewski $\mathrm{H}$, et al. Diagnostic and therapeutic gaps in patients with heart failure and chronic obstructive pulmonary disease. JACC Heart Fail. 2019;7(10):823-33.

5. Halaby R, Popma CJ, Cohen A, et al. D-Dimer elevation and adverse outcomes. J Thromb Thrombolysis. 2015; 39(1):55-9.

6. Adam SS, Key NS, Greenberg CS. D-dimer antigen: current concepts and future prospects. Blood. 2009; 113(13):2878-87.

7. Rodelo JR, De la Rosa G, Valencia ML, et al. D-dimer is a significant prognostic factor in patients with suspected infection and sepsis. Am J Emerg Med. 2012; 30(9):1991-9.

8. Zhou F, Yu T, Du R, et al. Clinical course and risk factors for mortality of adult inpatients with COVID19 in Wuhan, China: a retrospective cohort study. Lancet. 2020; 395(10229):1054-62. 
9. Tang N, Li D, Wang X, Sun Z. Abnormal coagulation parameters are associated with poor prognosis in patients with novel coronavirus pneumonia. J Thromb Haemost. 2020;18(4):844-7.

10. Agapakis DI, Tsantilas D, Psarris $P$, et al.Coagulation and inflammation biomarkers may help predict the severity of community-acquired pneumonia. Respirology. 2010; 15(5):796-803.

11. Zhang G, Zhang J, Wang B, Zhu X, Wang Q, Qiu S. Analysis of clinical characteristics and laboratory findings of 95 cases of 2019 novel coronavirus pneumonia in Wuhan, China: a retrospective analysis. Respir Res. 2020; 21(1):74.

12. Dai RX, Kong QH, Mao B, et al. The mortality risk factor of community acquired pneumonia patients with chronic obstructive pulmonary disease: a retrospective cohort study. BMC Pulm Med. 2018;18(1):12.

13. Tita-Nwa F, Bos A, Adjei A, Ershler WB, Longo DL, Ferrucci L. Correlates of D-dimer in older persons. Aging Clin Exp Res. 2010; 22(1): 20-3.

14. Douma RA, le Gal G, Söhne M, et al. Potential of an age adjusted D-dimer cut-off value to improve the exclusion of pulmonary embolism in older patients: a retrospective analysis of three large cohorts. BMJ. 2010; 340:c1475.

15. Vestbo J, Hurd SS, Agustí AG, et al. Global strategy for the diagnosis, management, and prevention of chronic obstructive pulmonary disease: GOLD executive summary. Am J Respir Crit Care Med. 2013; 187(4):347-65.

16. Ay C, Dunkler D, Pirker R, et al. High D-dimer levels are associated with poor prognosis in cancer patients. Haematologica. 2012; 97(8):1158-64.

17. Di Castelnuovo A, de Curtis A, Costanzo S, et al. Association of D-dimer levels with all-cause mortality in a healthy adult population: findings from the MOLI-SANI study. Haematologica. 2013; 98(9):147680.

18. Folsom AR, Delaney JA, Lutsey PL, et al. Associations of factor VIIIc, D-dimer, and plasminantiplasmin with incident cardiovascular disease and all-cause mortality. Am J Hematol. 2009; 84(6):349-53.

19. Cohen AT, Spiro TE, Spyropoulos AC, et al. D-dimer as a predictor of venous thromboembolism in acutely ill, hospitalized patients: a subanalysis of the randomized controlled MAGELLAN trial. J Thromb Haemost. 2014; 129(4): 479-87.

20. Wen D, Du X, Dong JZ, Zhou XL, Ma CS. Value of D-dimer and C reactive protein in predicting inhospital death in acute aortic dissection. Heart. 2013; 99(16):1192-7.

21. Ke L, Ni HB, Tong ZH, Li WQ, Li N, Li JS. D-dimer as a marker of severity in patients with severe acute pancreatitis. J Hepatobiliary Pancreat Sci. 2012; 19(3): 259-65.

22. Radenkovic $D$, Bajec $D$, Ivancevic $N$, et al. $D$-dimer in acute pancreatitis: a new approach for an early assessment of organ failure. Pancreas. 2009; 38(6):655-60.

23. Zorlu A, Yilmaz MB, Yucel H, Bektasoglu G, Refiker Ege M, Tandogan I. Increased D-dimer levels predict cardiovascular mortality in patients with systolic heart failure. J Thromb Thrombolysis. 2012; 33(4):322-8. 
24. Morange PE, Bickel C, Nicaud V, et al. Haemostatic factors and the risk of cardiovascular death in patients with coronary artery disease: the AtheroGene study. Arterioscler Thromb Vasc Biol. 2006; 26(12):2793-9.

25. Marcucci R, Gori AM, Giannotti F, et al. Markers of hypercoagulability and inflammation predict mortality in patients with heart failure. J Thromb Haemost. 2006; 4(5): 1017-22.

26. Querol-Ribelles JM, Tenias JM, Grau E, et al. Plasma D-dimer levels correlate with outcomes in patients with community-acquired pneumonia. Chest. 2004; 126(4):1087-92.

27. Shilon $Y$, Shitrit AB, Rudensky B, et al. A rapid quantitative $D$-dimer assay at admission correlates with the severity of community acquired pneumonia. Blood Coagul Fibrinolysis. 2003; 14(8):745-8.

28. Salluh JIF, Rabello LSCF, Rosolem MM, et al. The impact of coagulation parameters on the outcomes of patients with severe community-acquired pneumonia requiring intensive care unit admission. $\mathrm{J}$ Crit Care. 2011; 26(5):496-501.

29. Snijders D, Schoorl M, Schoorl M, Bartels PC, van der Werf TS, Boersma WG. D-dimer levels in assessing severity and clinical outcome in patients with community-acquired pneumonia. A secondary analysis of a randomised clinical trial. Eur J Intern Med. 2012; 23(5):436-41.

30. Shorr AF, Trotta RF, Alkins SA, Hanzel GS, Diehl LF. D-dimer assay predicts mortality in critically ill patients without disseminated intravascular coagulation or venous thromboembolic disease. Intensive Care Med. 1999; 25(2):207-10.

31. Yende S, D'Angelo G, Mayr F, et al. Elevated hemostasis markers after pneumonia increases one-year risk of all-cause and cardiovascular deaths. PLoS One. 2011; 6(8): e22847.

32. Chalmers JD, Singanayagam A, Scally C, Hill AT. Admission D-dimer can identify low-risk patients with community-acquired pneumonia. Ann Emerg Med. 2009; 53(5):633-8.

33. Clyne B, Olshaker JS. The C-reactive protein. J Emerg Med. 1999; 17(6): 1019-25.

34. Cuthbertson $\mathrm{CM}$, Christophi C. Disturbances of the microcirculation in acute pancreatitis. $\mathrm{Br} \mathrm{J}$ Surg. 2006; 93(5):518-30.

35. Smeeth L, Thomas SL, Hall AJ, Hubbard R, Farrington P, Vallance P. Risk of myocardial infarction and stroke after acute infection or vaccination. N Engl J Med. 2004; 351(25): 2611-18.

36. Corrales-Medina VF, Musher DM, Wells GA, Chirinos JA, Chen L, Fine MJ. Cardiac complications in patients with community acquired pneumonia: incidence, timing, risk factors, and association with short-term mortality. Circulation. 2012; 125(6): 773-81.

37. Kunter E, Ilvan A, Ozmen N, et al. Effect of corticosteroids on hemostasis and pulmonary arterial pressure during chronic obstructive pulmonary disease exacerbation. Respiration. 2008; 75(2):14554.

38. Ridker PM, Danielson E, Fonseca FA, et al. Rosuvastatin to prevent vascular events in men and women with elevated C-reactive protein. N Engl J Med. 2008; 359(21): 2195-207.

39. Zorlu A, Akkaya E, Altay $\mathrm{H}$, et al. The relationship between D-dimer level and the development of atrial fibrillation in patients with systolic heart failure. J Thromb Thrombolysis. 2012; 33(4):343-8. 
40. Unlü Y, Karapolat S, Karaca Y, Kiziltunç A. Comparison of levels of inflammatory markers and hemostatic factors in the patients with and without peripheral arterial disease. Thromb Res. 2006; 117(4):357-64.

41. Lindner G, Funk GC, Pfortmueller CA, et al. D-dimer to rule out pulmonary embolism in renal insufficiency. Am J Med. 2014; 127(4):343-7.

42. Shlipak MG, Fried LF, Crump C, et al. Elevations of inflammatory and procoagulant biomarkers in elderly persons with renal insufficiency. Circulation. 2003; 107(1):87-92.

43. Ariëns RA, de Lange $M$, Snieder $H$, Boothby $M$, Spector TD, Grant PJ. Activation markers of coagulation and fibrinolysis in twins: heritability of the prethrombotic state. Lancet. 2002; 359(9307): 667-71.

\section{Figures}

A

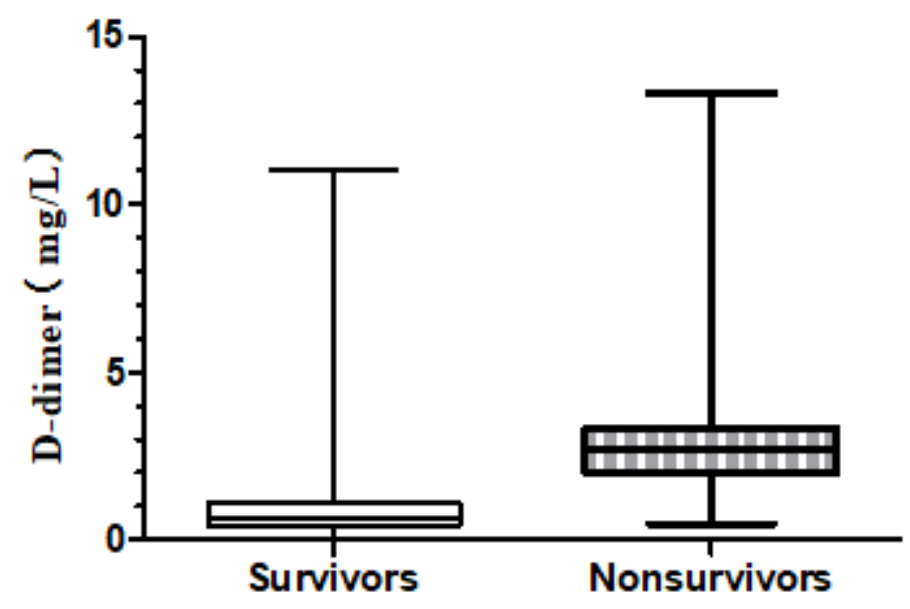

B

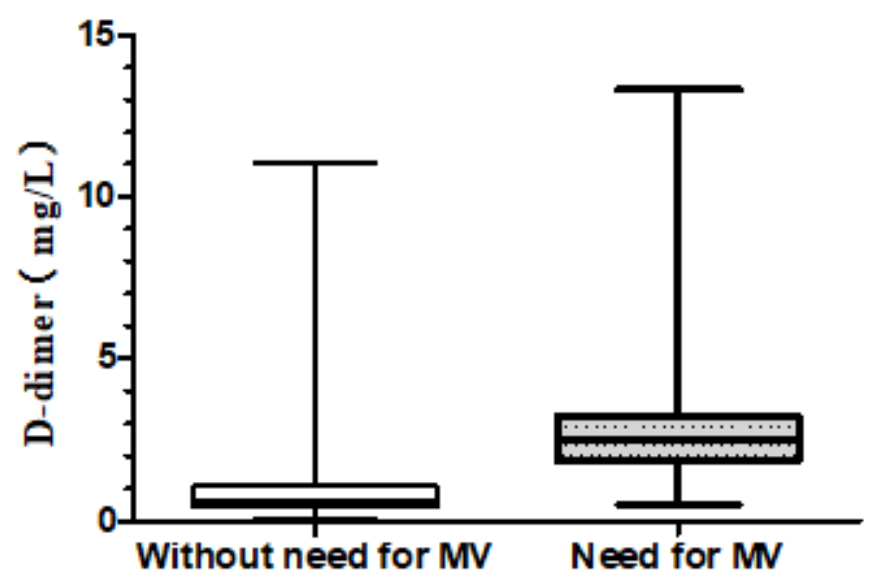

\section{Figure 1}

Differences in D-dimer levels between survivors and non survivors (A), and between need for MV and without need for MV (B). 


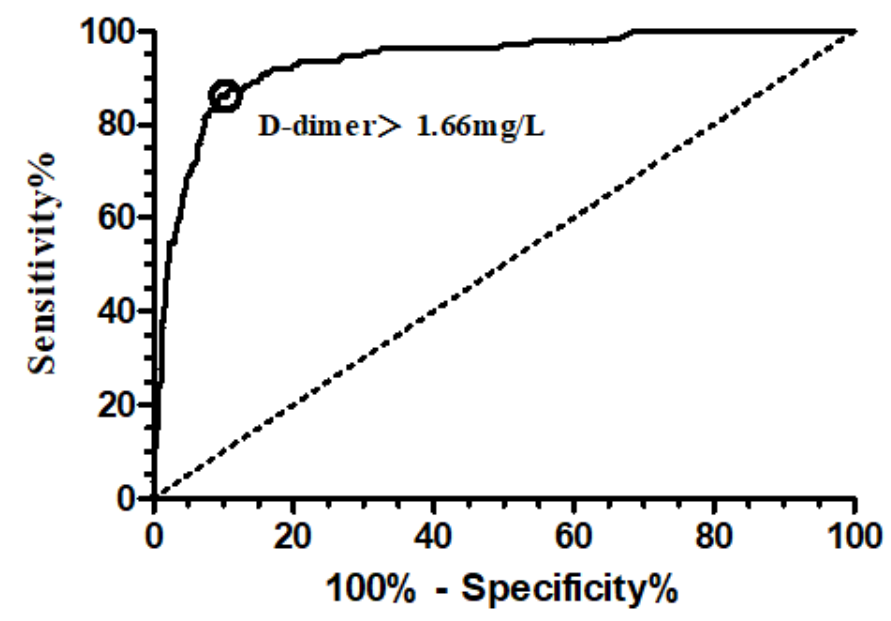

B

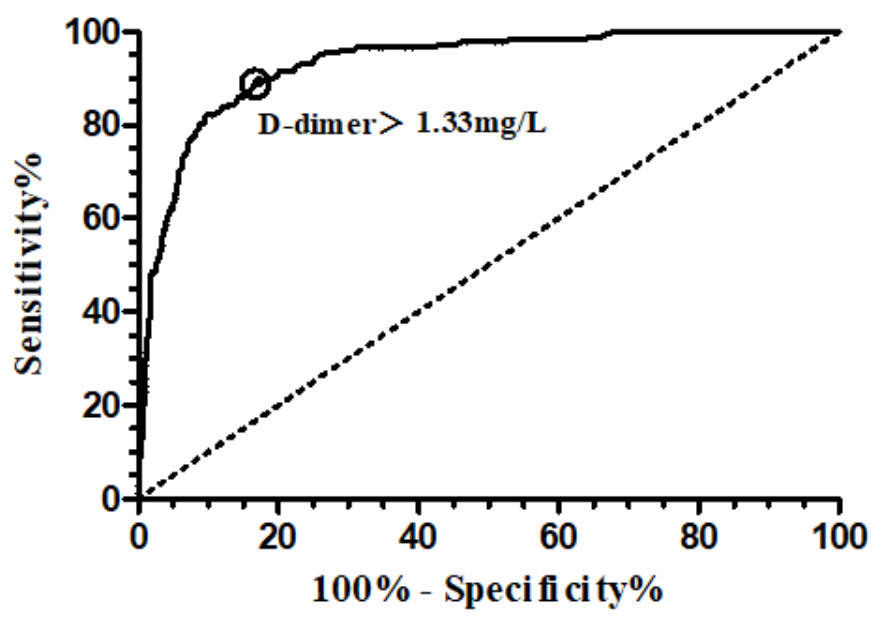

Figure 2

ROC curve for D-dimer as marker of 30-day mortality (A) and 30-day need for MV(B) in patients aged 80 years and older with AECOPD.

$\mathbf{A}$

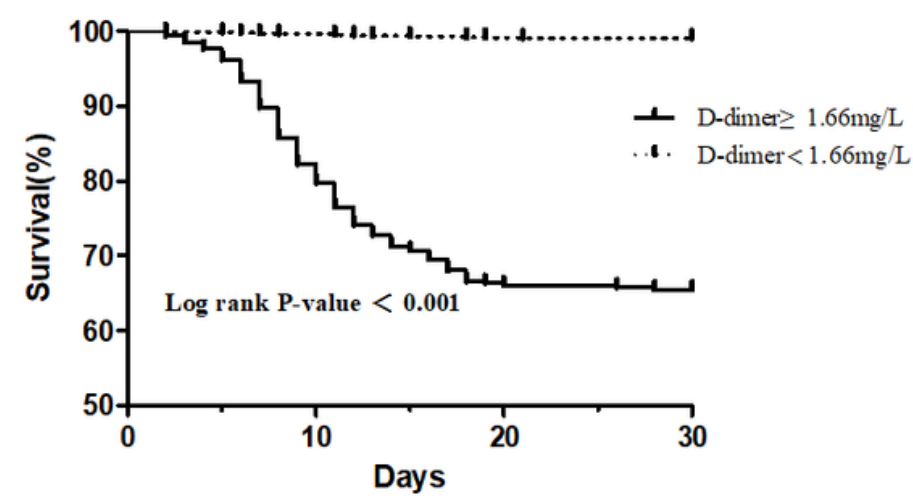

B

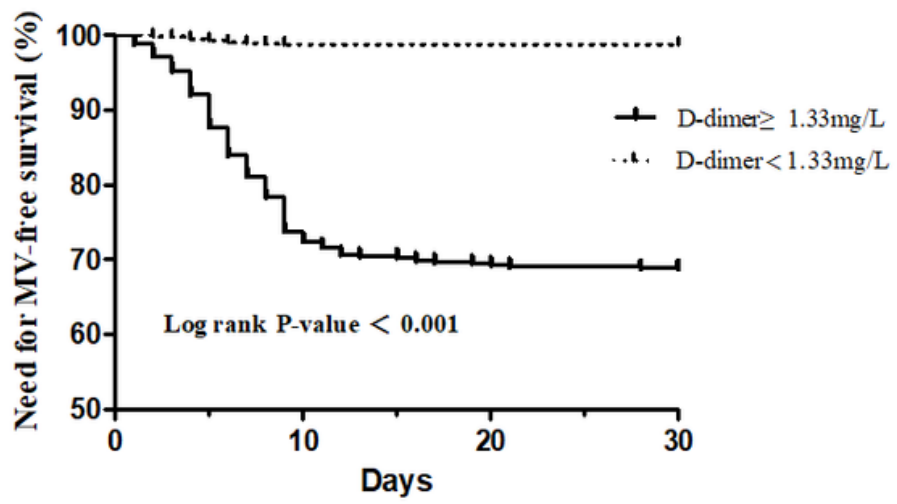

\section{Figure 3}

Kaplan-Meier survival curves (A) and need for MV-free survival curves (B) according to D-dimer levels. 